\title{
1 Supplements
}

3 Figure S1. Topography and location of the sampling site (a) location of Nanjing; (b)

4 location of surrounding cities of Nanjing; and (c) location of the sampling site at 5 Nanjing University. The map in (a) is from MeteoInfo developed by Yaqiang Wang;

6 the maps in (b) and (c) are reproduced from Google Maps (2021).

7

8

9 Figure S2. Temporal trend of the particle count of ${ }^{86}\left(\mathrm{C}_{2} \mathrm{H}_{5}\right)_{2} \mathrm{NCH}_{2}{ }^{+}$-containing 10 particles during the sampling period.

11

12

13

14

15

16

17

18

19

20

21

22

23

24

25

26

27

28

29 

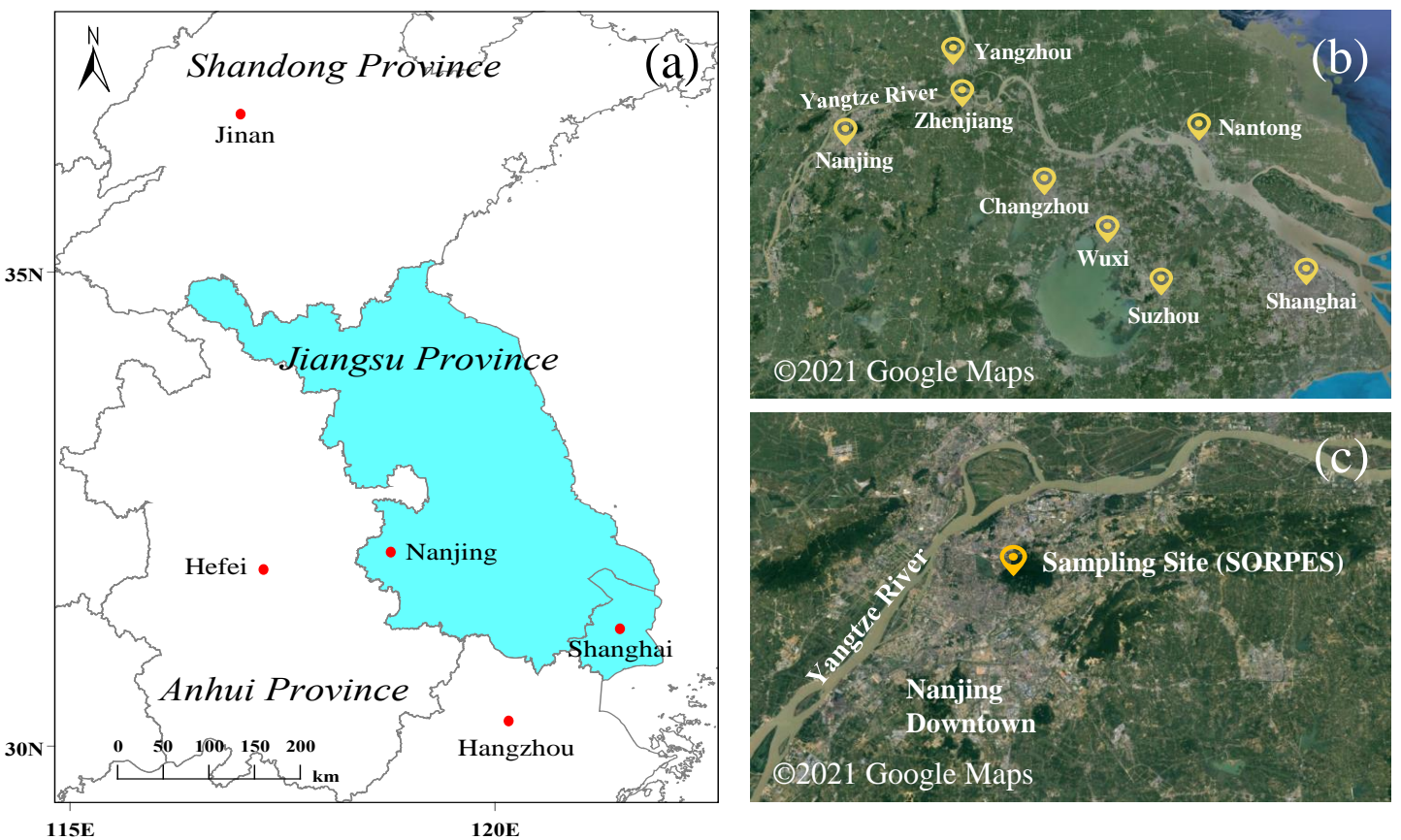

31 Figure S1. Topography and location of the sampling site (a) location of Nanjing; (b)

32 location of surrounding cities of Nanjing; and (c) location of the sampling site at

33 Nanjing University. The map in (a) is from MeteoInfo developed by Yaqiang Wang;

34 the maps in (b) and (c) are reproduced from Google Maps (2021).

35

36

37

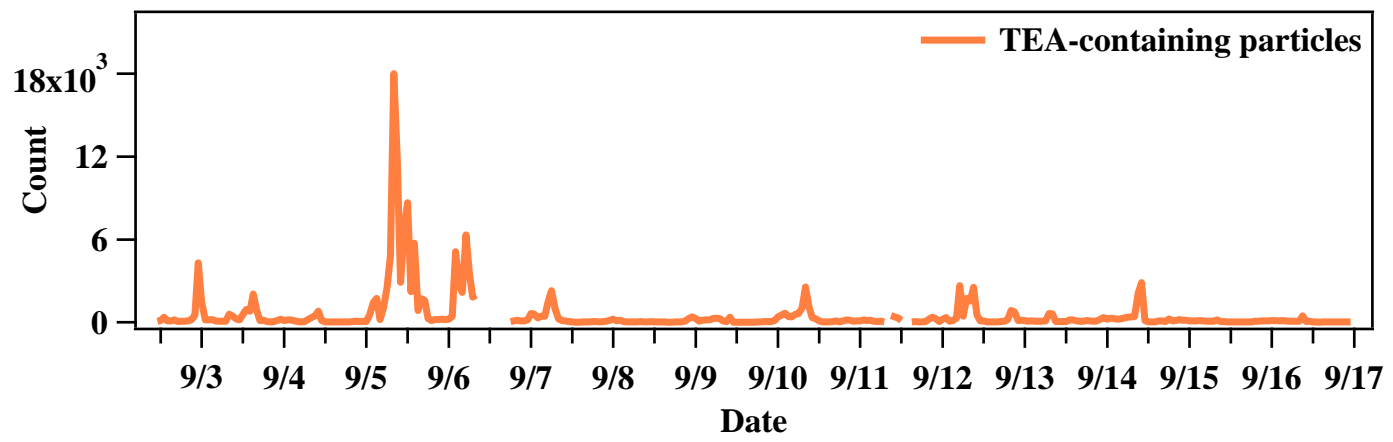

Figure S2. Temporal trend of the particle count of ${ }^{86}\left(\mathrm{C}_{2} \mathrm{H}_{5}\right)_{2} \mathrm{NCH}_{2}{ }^{+}$-containing 41 